\title{
Spatially Aware Tangible Display Interaction in a Tabletop Environment
}

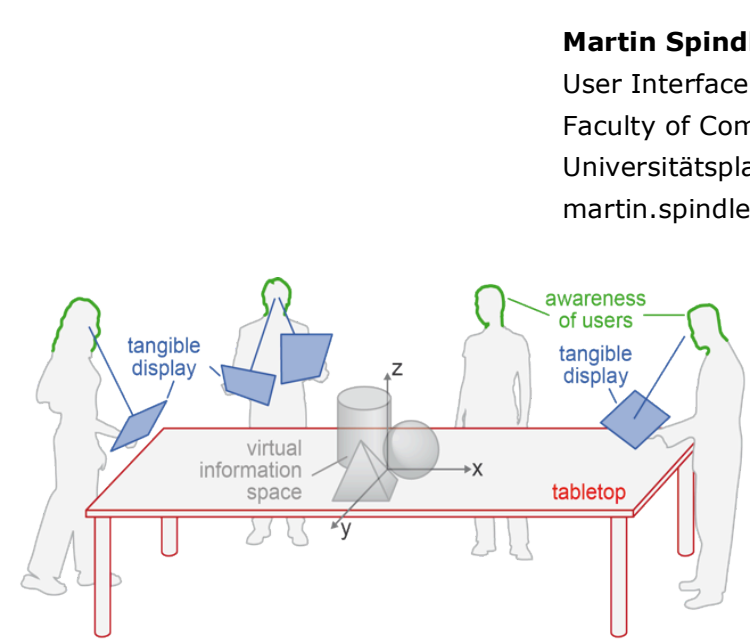

Figure 1. A multi-display tabletop environment consisting of following entities: a large tabletop, multiple spatially aware handheld displays, spatial awareness of users, virtual information spaces, and non-digital spatially aware objects.

\section{of interaction that allows users to interact} with complex information spaces in a more direct and natural way. The simultaneous use of multiple tangible displays explicitly supports collaborative work. My research includes the identification of basic interaction principles, the design and implementation of a technical framework, and the development and evaluation of interactive systems demonstrating the benefits of tangible displays for different application domains.

Copyright is held by the author/owner(s)

ITS'12, November 11-14, 2012, Cambridge, Massachusetts, USA ACM 978-1-4503-1209-7/12/11.

\section{Author Keywords}

Tangible magic lenses; PaperLens; digital tabletops; mobile displays; tangible interaction; user interfaces

\section{ACM Classification Keywords}

H.5.2. [Information interfaces and presentation]: User Interfaces: Input devices and strategies

\section{General Terms}

Human Factors, Design

\section{Introduction and Motivation}

Current technical developments in the smartphone and tablet market show that a fusion of input and output within the same device results in a more direct and natural interaction. While the majority of current approaches focuses on interacting on the surface of such mobile displays, e.g., by using multi-touch or pen input, interacting with handheld displays, i.e., by using their spatial relationships, still plays a rather minor role. When applied to a digital tabletop environment, the usage of handheld displays offers exciting new possibilities not only in terms of an enlarged presentation space but also as an increased interaction space. First, the tabletop display can act as a global view that can be shared by all users, whereas individual handheld displays can serve as personal views for single users. Thus the simultaneous use of multiple 


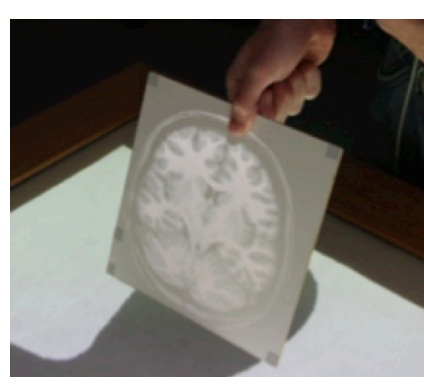

(a) Volume slicer

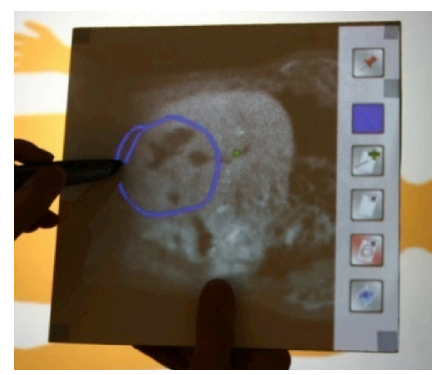

(b) Pen-based annotations

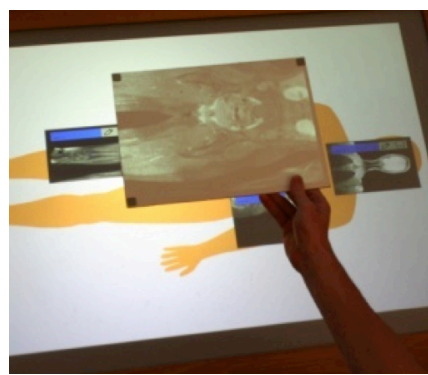

(c) Thumbnails on the table

Figure 2. Exploration of medical volumetric datasets with our tangible display system handheld displays can explicitly support collaborative work. Second, the spatial position and orientation of tangible displays, e.g., with reference to the table surface, can be used as a valuable form of input to the system. In this way, the previous $2 \mathrm{D}$ interaction space of the table display can effectively be extended to the physical 3D space above the table surface, thus better matching the volumetric character of complex 3D information spaces, such as medical MRI scans or 3D virtual environments. Third, tangible displays serve as a tool of representation (output) and interaction (input) at the same time. Fourth, the users' natural

understanding of spatial relationships between everyday objects can be leveraged towards a more seamless and natural interaction with complex information worlds by using tangible displays. Building and studying such multi-display tabletop environments is the subject of my dissertation research.

\section{Related Work}

Merging the digital world with the physical (analog) world is the vision of ubiquitous computing, as defined by Weiser [16]. This concept was adapted by Ishii's and Ullmer's tangible user interfaces (TUIs) [4], where interaction with digital information is provided through physical manipulation of real-world objects. Inspired by the notion of see-through interfaces [1], these objects can also be spatially aware handheld displays (e.g., mobile phones) that serve as tangible magic lenses into the virtual world. One of the first mobile displays for ubiquitous usage has been proposed by Fitzmaurice, who presented a spatially aware palmtop computer for exploring 3D-situated information spaces for revealing virtual information associated with arbitrary objects in an office environment [2]. With the metaDESK project, Ullmer and Ishii [15] applied this idea to a tabletop environment, where users can freely navigate through polygonal 3D models by moving an arm-mounted LCD display through the space above the tabletop that is also responsible for providing contextual graphica information.

In many situations, active display solutions, such as LCD panels, do not provide suitable form factors for a seamless integration into a tangible display system.

This is because active displays are often too heavy, too thick, too big, too rigid, and sometimes even too expensive if many devices are needed. This lack of technology motivated researchers to come up with a variety of lightweight display solutions that can easily be customized in terms of shape and size. Most of these solutions use a passive approach, i.e., image content is projected onto spatially aware projection screens in one or another form. Lee et al. [6], Holman et al. [3], and we [12] presented such systems that all use ceiling-mounted projectors for projecting dynamic video content onto optically tracked paper-like displays. SecondLight by Izadi et al. [5] is technically more complex in that it is based on electronically switchable diffusers. It supports dual back-projections on both a tabletop surface and tangible lenses above it. Another promising technological development are organic LEDs (OLED) that one day might unify the advantages of active and passive displays.

\section{Research Question \& Planned Contributions}

In my research I address the question of how to extend the interaction and representation space of digital tabletops into the third dimension by using the concept of spatially aware handheld displays. For this purpose, I propose a multi-display tabletop environment that supports following entities (see Figure 1): a large 


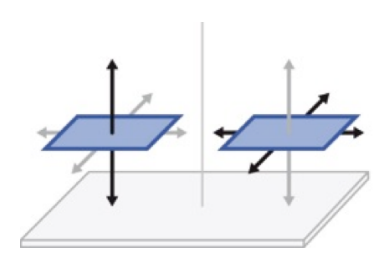

(a) Spatial position

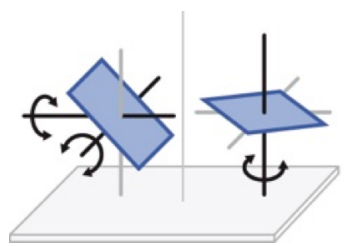

(b) Spatial orientation

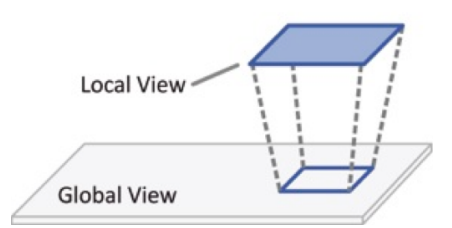

(c) Visual feedback

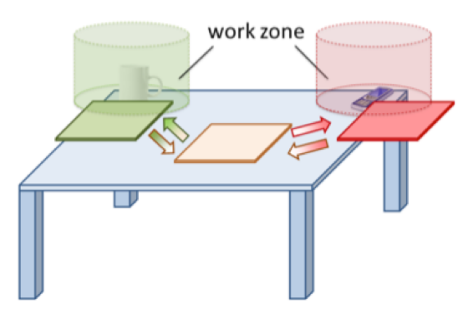

(d) Spatial work zones

Figure 3. Some of the interaction principles that we derived for tangible displays digital tabletop, multiple spatially aware handheld displays, the spatial awareness of users (e.g., head positions and orientations), virtual information spaces, and non-digital physical objects. I want to understand how such environments can leverage the user's natural understanding of spatial relationships of everyday objects aiming at making the overall interaction experience more seamless and natural. To address this research question, I plan to make the following three interdependent contributions:

(1) Conceptual Framework: My work will provide insights into the fundamental interaction principles a tangible display system should employ to meet the users' needs. These insights will be presented in the form of a conceptual framework and general design guidelines that unify different input modalities such as spatial relationships between displays, objects, and users as well as pen and multi-touch input. This includes the description of the main design variables, physical boundaries, and how they relate to tangible display interaction design.

(2) Technical Toolkit: The findings of Objective 1 will define technology requirements for designing and building a technical framework that make tangible display functionality accessible to developers. This includes tools for the spatial tracking of devices, the seamless communication between devices, and a programming framework for developing applications.

(3) Prototypes and Demonstrators: To evaluate and illustrate tangible display interaction principles (Objective 1), interactive prototypes and demonstrators need to be developed for different application domains. They will be built using the technical toolkit (Objective 2). Experiences with these systems will be used to refine the conceptual framework.

\section{Research Status: Conceptual Framework} With PaperLens [12], we developed the basic concept of tangible displays that employs the height and the horizontal position of handheld displays above the table as a means of interaction. This can be used, e.g., for zooming (by lifting/lowering a display) and panning (by horizontally moving a display) of large 2D pictures. We extended this concept and derived an interaction vocabulary [10] that describes essential dimensions for interacting with tangible displays. This includes spatial position and orientation (see Figure $3 a / b$ ), freezing, gestures (e.g., flipping and shaking), toolbox metaphor (e.g., the shape and size of displays), direct pointing (e.g., touch and pen based), visual feedback on the table (see Figure 3c), and the support of multiple displays. We also introduce the concept of spatial work zones (see Figure 3d) for pooling functionality, documents, and other digital content that is accessible by moving tangible displays into and through a work zone. In addition, we propose to leverage the spatial relationships between users and handheld displays, e.g., for the purpose of head-coupled perspective.

We distinguish between two basic groups of modes of how spatial movements can be interpreted. First, the system either can implicitly respond to positional changes of a tangible display or it can react only when the user explicitly says so, e.g., by pressing a button. Second, the interaction space associated with a particular tangible display either can move along with the display like a floating bubble that surrounds it or it can be fixed in space allowing the display to enter and leave the interaction space. 
One way of using the 3D space above a horizontal surface is to divide it into discrete parallel layers stacked upon each other. Horizontal and vertical display movements are essential tasks for the style of multilayer interaction associated with it. We conducted a comprehensive user study [8] with 18 participants investigating fundamental issues such as optimal number of layers and their thickness, movement and holding accuracies, and physical boundaries of the interaction volume.

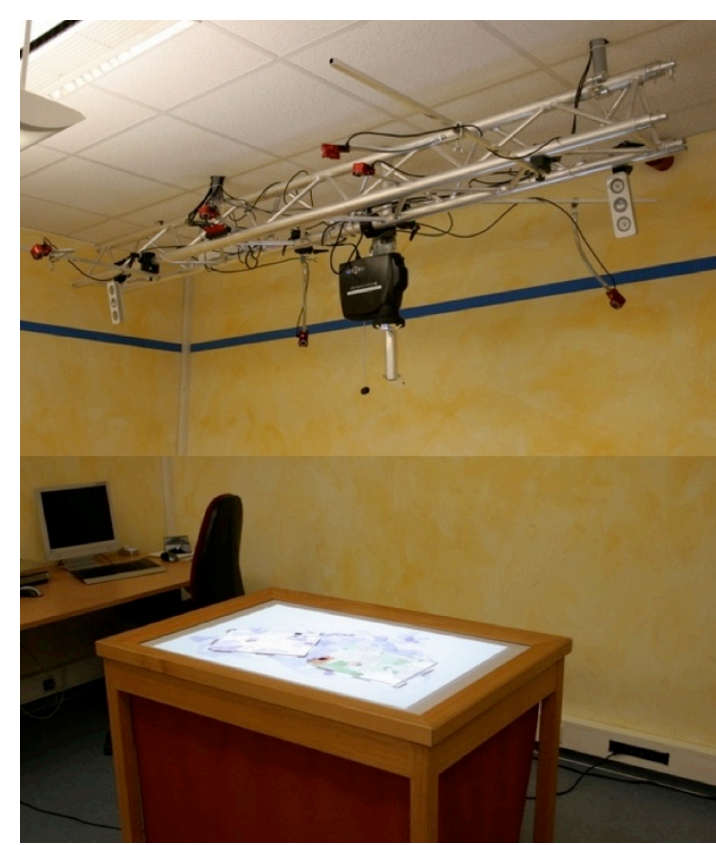

\section{Research Status: Technical Toolkit}

We decided on using a projective display approach as the technical basis for tangible displays. This gave us high flexibility in terms of form factors for the design of handheld displays - an important advantage for a research prototype. Examples for this are displaying content on the displays' backsides (required for flipping), arbitrarily shaped displays in different sizes (e.g., discs), the avoidance of display frames, and digital pen input [www.anoto.com].

Figure 4 shows the technical setup in our lab, see [12] and [10]. It consists of a backprojected tabletop, several infrared cameras, and a ceiling-mounted projector. The setup is enriched with various tangible displays made of cardboard in different sizes and shapes that can be freely moved

Figure 4. Technical setup of the tangible display system in our lab through the space on or above the tabletop. To bring such a system alive, several problems need to be solved: tracking of tangible displays, projecting image content onto them, recognizing gestures, support for direct pointing, and providing application functionality.
Many of these tasks can be tackled independently and thus have been split up between different computers. For the purpose of inter-computer communication, we use public protocols for streaming device states and remote procedure calls.

\section{Research Status: Demonstrators}

A multitude of application domains in fields like science, education, and entertainment can benefit from tangible display interaction principles. We developed several demonstrators illustrating and showcasing their use.

\section{Exploration of Scientific 3D Datasets}

One particular field of application for tangible displays is the exploration of large three-dimensional data. Besides geological or biological data, prime examples are medical volume datasets acquired from MRI or CT. With tangible displays in a tabletop environment, such data sets can be understood as residing in the space above the tabletop. The tabletop acts as a global view, providing reference for the users. For example, it can show an outline of the patient or a specific slice of the data set. The handheld displays provide local, personal views (see Figure $2 a$ ). When a user moves a display through the interaction volume, arbitrary, user-defined cutting planes can be computed in real-time and displayed, as showed by us in [12]. This form of direct interaction allows for fast and flexible exploration of the whole dataset or specific structures within. In addition, conspicuous structures can be annotated by using digital pen or finger based interaction techniques (see Figure $2 \mathrm{~b} / \mathrm{c}$ ), as demonstrated by us in [13].

\section{Information Visualization}

In many fields of scientific research, large amounts of data have to be visualized and examined. Such 


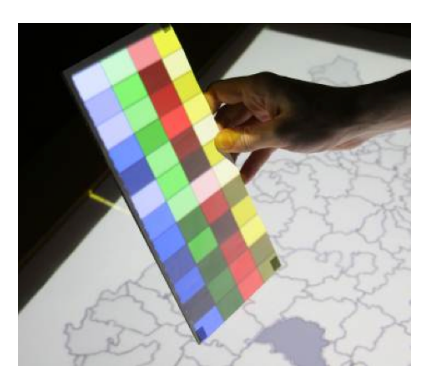

(a) Slicing the space-time-cube vertically to get an overview of time

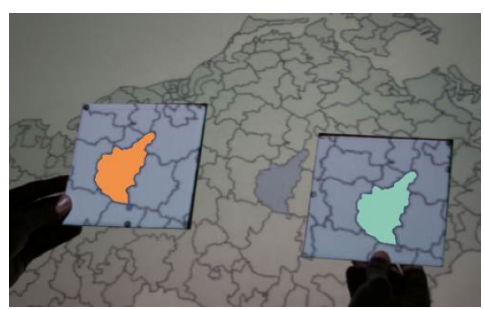

(b) Two-handed comparison

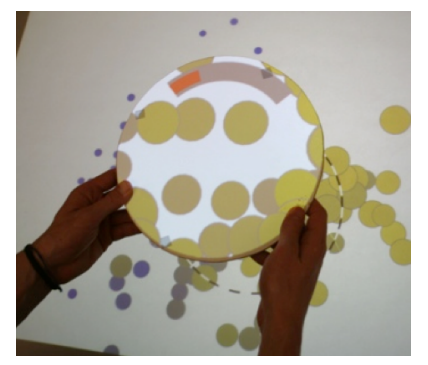

(c) Tangible fisheye lens

Figure 5. Tangible displays used for the purpose of information visualization complex datasets usually cannot be presented in a single image without the risk of cluttering. Filtering or presenting multiple views on the data can mitigate this problem. Tangible displays naturally support this by providing both additional display space and a new means for interaction, combined in single tangible objects, as described by us in [10].

One good example for this is space-time-cube visualization, which is an approach to integrate spatial and temporal aspects in a single visual representation. The analogy between a space-time-cube and the 3D presentation space of tangible displays motivated this idea: the tabletop shows the spatial context as a geographic map, and the dimension of time (e.g., 12 months) is mapped to the height above it. Tangible displays are used as physical viewports into the spacetime-cube. Interactive exploration is driven by horizontal and vertical translation to navigate the map and the time axis, respectively. When held in a horizontal orientation, a tangible view shows the data for a selected month, i.e., a horizontal slice through the space-time-cube. To get an overview for all months (i.e., a vertical slice), users can rotate a tangible view into upright orientation (see Figure 5a). The support for multiple independent handheld displays allows for collaboration between users or the visual comparison of portions of the data (see Figure $5 b$ ).

Beyond that, tangible displays support the focus and context concept. The tabletop serves as a reference, showing the contextual background in a main view. The handheld displays on the other hand provide additional physical display space that can be used to show local views into the information space. Here, the relative height of a tangible display may encode the zoom factor or, for a semantic zoom, a specific level of abstraction. Other examples include changing the parameters of a fisheye lens by rotating the display (see Figure 5c).

\section{Collected User Experiences}

We have given a number of demos of our tangible display system at international HCI conferences, e.g., at ACM ITS in Banff [14] and Saarbrücken [11], and we also frequently presented our system at public events at our local university. In total, several hundred visitors have tried out our prototypes, including average users, domain experts, and children. Overall, the feedback was very positive. Visitors appreciated how easy it was to learn the interaction techniques and to use the system. Since working with the system only requires users to grab one or more pieces of cardboard and move them through the space above the tabletop, which dynamically changes the image content displayed on them, a common comment was that the interaction was very natural and even felt somehow magical. Our experiences from qualitative users studies, e.g., [12], confirm this.

\section{Summary And Further Research}

A majority of research in the field of tangible user interfaces (TUI) focuses on input aspects by means of physical objects. In contrast, the goal of my dissertation is on making the output directly manipulable by using spatially aware displays. By allowing users to hold and move one or more lightweight handheld displays through the space above a tabletop, novel forms of interaction become possible providing a more natural and seamless way of interacting with complex information spaces, e.g., virtual 3D worlds [7] (see Figure 6). 


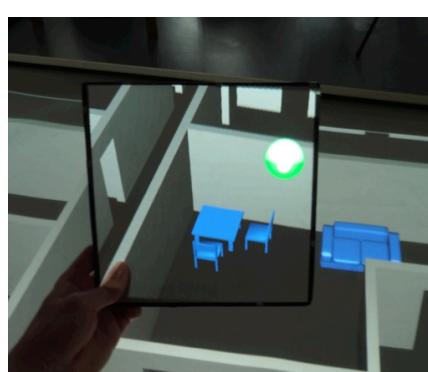

(a) A tangible peephole window

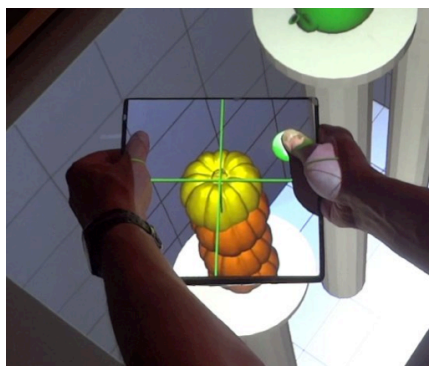

(b) Remote object selection

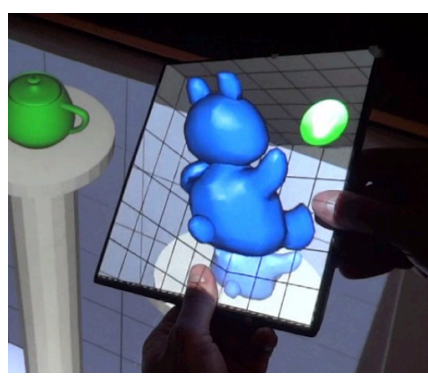

(c) Object inspection

Figure 6. Tangible displays combined with head-coupled perspectives (see [7])
My dissertation research is already in an advanced stage and I intend to submit and defend my thesis in 2013. In the future, lightweight organic displays (OLED) and simple spatial tracking solutions (e.g. optical depth sensors) are likely to replace the complex technical setup used for my work. This can help making the interaction techniques available for a broader audience one day. To take a first few steps towards this future, I am currently working on a less complex and easy to build technical setup [9] that is based on affordable consumer hardware, in my case the iPad and MS Kinect. Besides the future usage of lightweight smartphones and tablets as tangible displays, the interaction with digitally augmented everyday objects, such as coffee mugs, is another promising research objective for tangible displays.

\section{Acknowledgements}

My research is funded by the German Ministry of Education and Science (BMBF) project ViERforES-II (01IM10002B).

\section{References}

[1] E. A. Bier, M. C. Stone, K. Pier, W. Buxton, and T. D. DeRose. Toolglass and Magic Lenses: The See-Through Interface. Proc. SIGGRAPH 1993, ACM Press (1993), 445446 .

[2] G. W. Fitzmaurice. Situated Information Spaces and Spatially Aware Palmtop Computers, Communications of ACM, 36, 7 (1993), 39-49.

[3] D. Holman, R. Vertegaal, M. Altosaar, N. Troje, and D. Johns. Paper Windows: Interaction Techniques for Digital Paper. Proc. CHI '05, ACM Press (2005), 591-599.

[4] H. Ishii and B. Ullmer. Tangible Bits. Towards Seamless Interfaces between People, Bits and Atoms. Proc. CHI 1997, ACM Press (1997), 234-241.
[5] S. Izadi, S. Hodges, S. Taylor, D. Rosenfeld, N. Villar, A. Butler, and J. Westhues. Going Beyond the Display: A Surface Technology with an Electronically Switchable Diffuser. Proc. UIST '08, ACM Press (2008), 269-278.

[6] J. C. Lee, S. E. Hudson, J. W. Summet, and P. H. Dietz. Moveable Interactive Projected Displays Using Projector Based Tracking. Proc. UIST '05, ACM Press (2005), 63-72

[7] M. Spindler, W. Büschel, and R. Dachselt. Use Your Head: Tangible Windows for 3D Information Spaces in a Tabletop Environment. Proc. ITS '12, ACM Press (2012).

[8] M. Spindler, M. Martsch, and R. Dachselt. Going Beyond the Surface: Studying Multi-Layer Interaction Above the Tabletop. Proc. CHI '12, ACM Press (2012).

[9] M. Spindler, W. Büschel, and R. Dachselt. Towards Spatially Aware Tangible Displays for the Masses. Workshop on Designing Collaborative Interactive Spaces for e-Creativity, e-Science and e-Learning at AVI '12. ACM Press, 2012.

[10] M. Spindler, C. Tominski, H. Schumann, and R. Dachselt. Tangible Views for Information Visualization. Proc. ITS '10, ACM Press (2010), 157-166.

[11] M. Spindler, C. Tominski, M. Hauschild, H. Schumann and R. Dachselt. Novel Uses for Tangible Displays above the Tabletop. TechDemo at ITS ' 10.

[12] M. Spindler, S. Stellmach, and R. Dachselt. Advanced Magic Lens Interaction above the Tabletop. Proc. ITS '09, ACM Press (2009), 77-84.

[13] M. Spindler and R. Dachselt: Towards Pen-based Annotation Techniques for Tangible Magic Lenses Above a Tabletop. In Accompanying DVD of ACM ITS '09.

[14] M. Spindler and R. Dachselt. Advanced Magic Lens Interaction Above the Tabletop. TechDemo at ITS ' 09.

[15] B. Ullmer and H. Ishii. The metaDESK: Models and Prototypes for Tangible User Interfaces. Proc. UIST '97, ACM Press (1997), 223-232.

[16] M. Weiser. The Computer for the 21st Century. Scientific American. 265, 3 (1991), 66-75. 